\title{
Correlação entre pandemias: Tabagismo e a COVID-19
}

\author{
Correlation between pandemics: Smoking and COVID-19 \\ Correlación entre pandemias: Tabaquismo y COVID-19
}

\author{
Thais Carolina Alves Cardoso \\ ORCID: https://orcid.org/0000-0002-0209-3840 \\ Universidade Evangélica de Goiás UniEvangélica, Brasil \\ E-mail: thaisscarolinaa@hotmail.com \\ Adriano Ferro Rotondano Filho \\ ORCID: https://orcid.org/0000-0002-8176-6432 \\ Universidade Evangélica de Goiás UniEvangélica, Brasil \\ E-mail: adrianoferro09@gmail.com \\ Jalsi Tacon Arruda \\ ORCID: https://orcid.org/0000-0001-7091-4850 \\ Universidade Evangélica de Goiás UniEvangélica, Brasil \\ E-mail: jalsitacon@gmail.com
}

\begin{abstract}
Resumo
O hábito de fumar ou tabagismo, é algo comum desde os primórdios da humanidade. Compartilhar qualquer uma das diferentes formas de consumo de tabaco aumentam a via de transmissão do SARS-CoV-2. Além disso, a pandemia da COVID-19 tornou obrigatório o distanciamento social, muitas famílias dentro de casa, obrigou pessoas que não fumam a conviver com fumantes, tornando-as mais vulneráveis ao tabagismo passivo e, consequentemente a gravidade da COVID-19. O presente estudo tem como objetivo evidenciar a relação entre o tabagismo e a COVID-19. Foi realizada uma revisão integrativa de literatura a partir de estudos originais obtidos nas bases de dados SciELO, PubMed, Google Acadêmico e LILACS. O tabagismo e a COVID-19 são duas pandemias que podem ser associadas. O hábito de fumar se constitui como o principal fator de risco para doenças e infecções respiratórias graves, devido as mudanças estruturais no trato respiratório e a redução da resposta imune. É muito importante monitorar a iniciação do tabagismo, principalmente entre os jovens, por essa ser uma ação passível de prevenção. O tabagismo é um hábito que contribui para a transmissão, infecção e agravamento da COVID-19.
\end{abstract}

Palavras-chave: Hábito de fumar; Adolescente; COVID-19.

\begin{abstract}
The habit of smoking or smoking is something common since the dawn of humanity. Sharing any of the different forms of tobacco use increases the transmission pathway for SARS-CoV-2. In addition, the COVID-19 pandemic made social distancing mandatory, many families at home, forced people who do not smoke to live with smokers, making them more vulnerable to passive smoking and, consequently, the severity of COVID-19. The present study aims to highlight the relationship between smoking and COVID-19. An integrative literature review was carried out based on original studies obtained from the SciELO, PubMed, Academic Google, and LILACS databases. Smoking and COVID-19 are two pandemics that can be associated. Smoking is the main risk factor for severe respiratory diseases and infections, due to structural changes in the respiratory tract and reduced immune response. It is very important to monitor smoking initiation, especially among young people, as this is a preventable action. Smoking is a habit that contributes to the transmission, infection, and aggravation of COVID-19.
\end{abstract}

Keywords: Smoking; Adolescent; COVID-19.

\section{Resumen}

El hábito de fumar o fumar es algo común desde los albores de la humanidad. Compartir cualquiera de las diferentes formas de consumo de tabaco aumenta la vía de transmisión del SARS-CoV-2. Además, la pandemia COVID-19 hizo obligatorio el distanciamiento social, muchas familias en el hogar, obligó a las personas que no fuman a vivir con fumadores, haciéndolos más vulnerables al tabaquismo pasivo y, en consecuencia, a la gravedad del COVID-19. El presente estudio tiene como objetivo resaltar la relación entre el tabaquismo y COVID-19. Se realizó una revisión integradora de la literatura a partir de estudios originales obtenidos de las bases de datos SciELO, PubMed, Google Académico y LILACS. El tabaquismo y el COVID-19 son dos pandemias que pueden estar asociadas. El tabaquismo es el principal factor de riesgo de enfermedades e infecciones respiratorias graves, debido a los cambios estructurales en el tracto respiratorio y la respuesta inmunitaria reducida. Es muy importante vigilar la iniciación al tabaquismo, especialmente entre los jóvenes, ya que se trata de una acción prevenible. Fumar es un hábito que contribuye a la transmisión, infección y agravamiento del COVID-19.

Palabras clave: Fumar; Adolescente; COVID-19. 


\section{Introdução}

Fumar é algo comum desde os primórdios da humanidade. O consumo do tabaco teve início no final do século XIX, impulsionado pela invenção da máquina de fabricar cigarros elevou o consumo chegando a uma pandemia do fumo. Já no século XX o hábito de fumar passou a ser influenciado pela indústria da propaganda, o cinema, as grandes guerras e a ampliação da circulação de mercadorias e de pessoas (Cardoso et al., 2021). O tabagismo apresenta evolução crônica e mata mais de 8 milhões de pessoas por ano, deste modo o ato de fumar gera extenso comprometimento à saúde do indivíduo, tornando-o mais susceptível a infecções (Pessoa et al., 2020). O cigarro possui mais de 4.800 substâncias tóxicas que provocam inúmeras morbidades, contudo, a dependência química expõe o tabagista a diversas vulnerabilidades gerando agravos que evoluem para doenças crônicas como câncer, doenças cardiovasculares e pulmonares (Rodrigues et al., 2020).

O hábito de fumar apresenta variações, mas é uma importante causa de perda de saúde. Além disso, está associado ao desenvolvimento de uma série de doenças com mortalidade geral duas vezes maior nos fumantes se comparado aos nãofumantes. Ainda assim, apesar de ser um importante fator de risco e, cuja remoção repercute significativamente na redução da morbimortalidade, os dados associados a prevalência de tabagismo na população brasileira são ainda esparsos, apesar dos inúmeros estudos sobre o tema. As estimativas oscilam entre $32 \%$ e $42 \%$ da população, sendo maioria dos fumantes homens entre a $3^{\mathrm{a}}$ e $6^{\mathrm{a}}$ décadas de vida e posição socioeconômica inferior (Costa et al., 2020; Cardoso et al., 2021).

Diante do novo coronavírus identificado pela primeira vez em Wuhan, na China, em dezembro de 2019, com a rápida disseminação e susceptibilidade e a gravidade da doença tornaram essa infecção uma das maiores crises globais enfrentadas nos últimos tempos. A proporção de pessoas contaminadas em todo o mundo foi tamanha que em 11 de março de 2020 a Organização Mundial de Saúde (OMS) declarou a COVID-19 (coronavirus disease 2019) como foi nomeada a doença ocasionada pelo vírus SARS-CoV-2, como uma pandemia. Apesar da maioria das pessoas infectadas apresentar sintomas leves, como tosse seca, febre e cansaço, algumas pessoas podem ter o agravamento do quadro clínico, necessitando o uso de suporte ventilatório (OPAS, 2020; Salem Szklo, 2020; WHO, 2020a).

A pandemia da COVID-19 pode ser recente e ter mobilizado inúmeros esforços dos governos para combatê-la, mas existe uma pandemia muito mais antiga e com evolução crônica: a do uso do tabaco, que, apesar de todo conhecimento a seu respeito, campanhas e estratégias para prevenção e cessação, ainda mata milhares de pessoas por ano (INCA, 2020; Cavalcante et al., 2020a). De acordo com a avaliação da OMS, na década de 1990-1999, 3 a 4 milhões de pessoas no mundo morreram anualmente de doenças induzidas pelo uso de tabaco. Em outra abordagem relacionada ao tema, o Programa Nacional de Controle do Tabaco, do Ministério da Saúde Pública destacou que se trata de uma situação que afeta fundamentalmente os países em desenvolvimento, e que somente no ano 2000, 4,8 milhões morreram por doenças relacionadas a esse hábito, sendo 3,8 milhões de homens e 1 milhão de mulheres. Estima-se que em 2025 esse número possa aumentar para 10 milhões de indivíduos (González Hidalgo et al., 2012; WHO, 2020b).

Apesar do número de fumantes ter reduzido nos últimos anos, cerca de 20 milhões de pessoas ainda fumam no Brasil, e, pela dependência de nicotina se expõem a inúmeras outras substâncias tóxicas contidas no tabaco, ficando mais vulneráveis a doenças graves que, atualmente, constituem-se como fatores de risco para o agravamento da infecção por COVID-19 (Cavalcante et al., 2020b). O uso do narguilé também pode aumentar o risco da transmissão do vírus, pelo compartilhamento de bocais e mangueiras. Além disso, a OMS alerta que os fumantes possuem maior risco de contrair a infecção do novo coronavírus uma vez que levam a mão à boca por diversas vezes no ato de fumar (Rodrigues et al., 2020).

A pandemia da COVID-19 também tornou obrigatório o distanciamento social, fazendo com que muitas famílias passem mais tempo dentro de suas casas. Assim, as pessoas que não fumam, mas convivem com fumantes, tornam-se mais vulneráveis a exposição excessiva à fumaça e às substâncias tóxicas contidas no tabaco e consequentemente a gravidade da infecção pela COVID-19 (INCA, 2020; Melo et al., 2020). Dessa forma, o tabagismo e a COVID-19 são duas pandemias 
associadas. Todas as formas de consumo de tabaco aumentam o risco para COVID-19, inclusive a forma mais crítica que pode levar a um desfecho fatal. Compartilhar qualquer produto de tabaco é via de transmissão do SARS-CoV-2. Além disso, o consumo de cigarros convencionais, cigarros eletrônicos e tabaco aquecido, em portadores assintomáticos contribui para disseminar a COVID-19 (Araújo, 2020; WHO, 2020b). Diante do exposto, esse estudo tem como objetivo evidenciar a relação entre o tabagismo com a infecção pelo SARS-CoV-2 que ocasiona a COVID-19.

\section{Metodologia}

O presente estudo enquadra-se nas definições de uma revisão integrativa da literatura que tem por finalidade sintetizar resultados obtidos em pesquisas, de maneira ordenada e abrangente, integrando o conhecimento sobre um determinado tema. Foi realizada em seis etapas: identificação do tema, estabelecimento dos critérios de inclusão e exclusão, busca e identificação dos estudos, categorização dos estudos selecionados, análise e interpretação dos dados obtidos e apresentação da revisão/síntese dos conhecimentos.

Para a obtenção dos estudos as buscas foram realizadas nas bases de dados Scientific Electronic Library Online (SciELO), Public Scientific (PubMed), Google Acadêmico e Literatura Latino-Americana e do Caribe em Ciências da Saúde (LILACS). Foram utilizados os seguintes descritores Ciências da Saúde (DeCS), que constam na plataforma DeCS da Biblioteca Virtual de Saúde (BVS): "Fumar" e "COVID-19”, utilizados em português, inglês e espanhol, que foram utilizados em combinação, com auxílio de operadores Booleanos (AND/OR). A coleta de dados foi realizada entre março e julho de 2021. A escolha dessas bases ocorreu em razão da amplitude no que tange à abrangência dos periódicos indexados que atuam na temática discutida. Os estudos incluídos na revisão integrativa permitem uma avaliação crítica do tema discutido sendo possível identificar lacunas que poderão direcionar futuras pesquisas.

Os critérios de inclusão foram: artigos originais ou de revisão e relatos de casos disponíveis completos que investigaram a relação do tabagismo com a infecção pela COVID-19; sem restrição de data de publicação e publicados nos idiomas português, inglês e espanhol. Foram excluídos estudos que não investigassem o tema proposto, artigos de opinião, carta ao editor, e que não atendessem aos critérios de inclusão descritos. A primeira etapa de seleção foi identificar os estudos e se preenchiam os critérios estabelecidos, passando por uma seleção inicial das publicações realizada com leitura do título e resumo para verificar se enquadravam na temática. Após essa etapa, os estudos previamente selecionados foram lidos na íntegra, empregando os critérios de inclusão e exclusão, para análise e obtenção das informações necessárias para a construção do presente estudo. Caso houvesse discordância entre os avaliadores sobre os critérios analisados, foi realizada discussão específica sobre o artigo em questão, confrontando ideias com base nos critérios adotados para a revisão.

\section{Resultados e Discussão}

Reconhecido como uma doença crônica, o hábito de fumar ou tabagismo tem como causa a dependência de nicotina, um alcaloide básico psicoativo encontrado em produtos feitos à base de tabaco. A nicotina exerce dois efeitos: estimulante no locus ceruleus e um efeito de recompensa no sistema límbico, altamente viciante. Quando a nicotina é consumida através do tabaco (da planta Nicotiana tabacum) apresenta um efeito estimulante e tranquilizante após algumas tragadas, com efeito bloqueador do estresse (Cardoso et al., 2021). O hábito de fumar se constitui como o principal fator de risco para doenças e infecções respiratórias graves, devido as mudanças estruturais no trato respiratório e a redução de sua resposta imune (Piras et al., 2020; Castedo et al., 2021). Com isso, portanto, o tabagismo é considerado um grande causador de adoecimento e morte em nível mundial. E a Classificação Estatística Internacional de Doenças e Problemas Relacionados à Saúde (CID-10) qualifica o tabagismo como integrante do grupo de transtornos mentais e comportamentais motivado pelo uso de substância psicoativa (INCA, 2020). 
Durante a pandemia da COVID-19 a questão do tabagismo e o risco de infecção respiratória aguda são tópicos muito discutidos. O foco na prevenção e cessação do tabagismo concentra-se nas mortes não infecciosas respiratórias, cardiovasculares e relacionadas ao câncer, e grande parte da retórica promocional do cigarro eletrônico gira em torno de potencialmente salvar bilhões de vidas que poderiam ser perdidas devido a resultados não infecciosos (Faria \& Fonseca, 2021). O risco de complicações infecciosas é, no entanto, o foco e preocupação predominante em países de baixa e média renda, particularmente durante pandemias (van Zyl-Smit et al., 2020). Os riscos de complicações pela COVID-19 são aplicáveis também aos usuários de narguilés, cigarros eletrônicos (e-cigs, vape ou vaping devices) e do tabaco aquecido, pelo fato de que o usuário exala gotículas de vapor e propaga o SARS-CoV-2 (Piras et al., 2020; Castedo et al., 2021).

Diante disso, fumantes fazem parte do grupo de risco para a contaminação pela COVID-19. Além da possibilidade de inferir sobre o aumento do risco de contaminação desse grupo na medida em que fumantes levam os produtos de tabaco, quer sejam tradicionais ou dispositivos eletrônicos para fumar (DEF) a boca, sem a adequada higienização das mãos. Os narguilés, muito populares entre os jovens, que geralmente compartilha os bocais, também facilitam a transmissão do vírus. A OMS demonstrou preocupação com o potencial para a disseminação da doença por intermédio do uso dos produtos de tabaco. Portanto, por suas características que possibilitam seu compartilhamento, os DEFs também devem ser incluídos como produtos que contribuem para a contaminação pela síndrome respiratória aguda grave provocada pelo coronavírus 2 (SARS-CoV-2) (Silva et al., 2020).

De acordo com o estudo realizado por Sokolovsky et al. (2021), em que examinaram as mudanças no uso do tabaco em estudantes universitários jovens adultos no contexto da pandemia COVID-19, com foco no fumo e na vaporização, foi observado que maior ansiedade e mudança de casa (versus viver de forma independente) estava relacionada a maior chance de pausa no hábito; enquanto COVID-19 relacionada a exposição e busca por notícias foram relacionadas a menores chances de pausar esse hábito ruim. Em um estudo realizado na Inglaterra, após o lockdown promovido pela COVID-19 durante um período do ano de 2021, observou-se que esse momento não foi associado a uma mudança significativa na prevalência de tabagismo (17\% depois X 15,9\% antes do lockdown), mas foi associado com aumentos nas tentativas de abandono (39,6 versus $29,1 \%)$, sucesso de abandono (21,3 versus $13,9 \%)$ e cessação $(8,8$ versus $4,1 \%)$ entre os fumantes em 2020 (Jackson et al., 2021).

Além do tabagismo predispor a diversas doenças pode ser considerado como um fator de risco para o aumento da gravidade da COVID-19 (Silva et al., 2020; Komiyama \& Hasegawa, 2020). De acordo com o estudo realizado por Emami et al. (2020), entre os pacientes hospitalizados com COVID-19, as doenças de base que mais se destacam incluem o tabagismo. Esse fator pode ser observado no resumo científico realizado pela OMS, com 34 artigos apontando que 26 estudos observacionais foi relatado a prevalência do tabagismo entre os pacientes internados com o novo coronavírus (WHO, 2020a).

Os fumantes, ex-fumantes e portadores de Doença Pulmonar Obstrutiva Crônica (DPOC) por apresentarem aumento da expressão da enzima conversora de angiotensina tipo 2 (ECA-2) nas vias aéreas inferiores, são um grupo de risco para a infecção e complicações da COVID-19, tanto pelas comorbidades tabaco-relacionadas, quanto pelos elevados níveis de proteína-C reativa (PCR), D-Dímero e das citocinas pró-inflamatórias. Isto infere que fumar contribui para o aumento dos receptores virais sendo, portanto, importante fator de risco para complicações e prognóstico reservado na COVID-19. Entre os pacientes infectados, os fumantes têm um risco duas vezes maior de serem internados em unidade de terapia intensiva (UTI), de necessitar de ventilação mecânica e de óbito do que os pacientes não fumantes (Araújo, 2020; Cavalcante et al., 2020b; Scholz et al., 2020). ECA-2 foi identificado como um receptor de entrada para o coronavírus em células humanas, incluindo o SARS-CoV-2 que causa a COVID-19 (Hamet et al., 2021).

Liu et al. (2020), realizaram um estudo de série de casos retrospectivo, em um único local, de associações clínicas entre achados epidemiológicos e manifestações clínicas, resultados radiográficos ou laboratoriais. Esse estudo foi caracterizado 
por 954 pacientes, incluindo 56 com história de tabagismo, a história de tabagismo aumentou o risco de COVID-19 grave com uma razão de chances de 5,5. A análise semiquantitativa das imagens do pulmão para cada um dos cinco lobos revelou uma diferença significativa nem na lesão pulmonar no primeiro exame, nem na dinâmica da lesão pulmonar em diferentes momentos de exames entre os grupos de fumantes e não fumantes. Nenhuma diferença significativa foi encontrada nos resultados laboratoriais, incluindo dímero $\mathrm{D}$ e níveis de proteína $\mathrm{C}$ reativa, exceto covariâncias diferentes para densidade de linfócitos de células imunes e neutrófilos.

Em um estudo realizado por Gülsen et al. (2021), cujo objetivo foi determinar a relação entre o tabagismo e a gravidade clínica da COVID-19 por meio de uma metanálise sistemática. O grupo de estudo consistia em um total de 16 artigos, em que 11.322 pacientes com COVID-19 foram incluídos. Esse estudo revelou uma relação de 2,17 entre história de tabagismo e casos graves de COVID-19. Além disso, encontrou-se uma associação de 1,51 entre o tabagismo atual e COVID19 grave. Em 10,7\% dos não fumantes foram casos graves de COVID-19, enquanto em fumantes ativos casos graves ocorreram em $21,2 \%$.

Outro estudo realizado em Wuhan investigou os fatores que afetavam a progressão da pneumonia em pacientes com COVID-19. O grupo de estudo foi constituído por pacientes internados com diagnóstico positivo para o SARS-CoV-2 (30/12/2019 a 15/01/2020), totalizando 78 pacientes. Após duas semanas, 14\% destes pacientes tiveram piora do quadro clínico ao passo que $86 \%$ deles estabilizaram. Dentre os pacientes que apresentaram agravo na evolução da doença $(\mathrm{n}=11)$ cerca de $27 \%$ tinham histórico de tabagismo em detrimento a $3 \%$ dos pacientes do grupo que estabilizou. Os autores concluíram que a proporção de pacientes com histórico de tabagismo foi maior no grupo com piora na evolução da doença em comparação ao grupo que estabilizou e rapidamente evoluiu para a melhora (Liu et al., 2020). Tais achados sugerem estar o tabagismo associado à má progressão da infecção pelo COVID-19 (Silva et al., 2020).

Pode-se inferir, portanto, que o aumento nas taxas de cessação do uso de tabaco poderia impactar a transmissão comunitária de SARS-CoV-2. Em epidemias virais anteriores, houve evidências de que abordagens multifacetadas para a cessação do tabagismo, através de intervenções comportamentais e farmacológicas, podem ter um papel significativo em ambas as situações, epidemia viral e tabagismo (Faria \& Fonseca, 2021). A cessação do tabagismo, com o passar do tempo, leva a normalização de parte da arquitetura do epitélio respiratório, com diminuição da hiperplasia e downregulation dos níveis de ECA-2 (Chatkin \& Godoy, 2020).

\section{Conclusão}

O tabagismo constitui-se de um hábito contribuinte importante para a transmissão, infecção e agravamento da COVID-19. Neste contexto é fundamental trabalhar com os tabagistas a cessação do uso para reduzir os agravos que os fumantes podem sofrer mediante uma infecção e, assim, melhorar o prognóstico do paciente que seja contaminado pelo SARCS-CoV-2. A redução do número de tabagistas pode, portanto, contribuir para diminuir a possibilidade de curso grave da infecção pelo novo Coronavírus.

\section{Referências}

Araújo, A. J. de (2020). A dependência do tabaco na pandemia e a relevância da associação COVID-19 e tabaco. 29(1), 28-31.

Cardoso, T. C. A., Rotondano Filho, A. F., Dias, L. M., \& Arruda, J. T. (2021). Aspectos associados ao tabagismo e os efeitos sobre a saúde. Research, Society and Development, 10(3), e11210312975. 10.33448/rsd-v10i3.12975

Castedo, C. R., Signes-Costa, J., \& Jiménez-Ruiz, C. A. (2021). COVID-19 y tabaco. Archivos de Bronconeumologia, 57, 5. 10.1016/j.arbres.2020.07.014

Cavalcante, T. M., Lacerda Mendes, F., de Abreu Perez, C., Ribeiro de Carvalho, A. O., Leal Teixeira, A. P., \& Rodrigues Viegas, J. R. (2020a). Como a Política Nacional de Controle do Tabaco Pode Ajudar no Enfrentamento da Pandemia da Covid-19? Revista Brasileira de Cancerologia, 66(TemaAtual), e1023. https://doi.org/10.32635/2176-9745.RBC.2020v66nTemaAtual.1023 
Cavalcante, T. M., Perez, C. de A., Mendes, F. L., Machado, A. T., Rangel, E. C., Pereira, R. C. A., \& de Miranda Leal, A. M. (2020b). Covid-19 e tabagismo: aspectos epidemiológicos, biológicos, psicossociais e implicações para a Política Nacional de Controle do Tabaco. Revista Brasileira de Cancerologia, 66 (Tema Atual), e-1039. https://doi.org/10.32635/2176-9745.RBC.2020v66nTemaAtual.1039

Chatkin, J. M., \& Godoy, I. (2020). Are smoking, environmental pollution, and weather conditions risk factors for COVID-19? Jornal Brasileiro de Pneumologia, 46(5):e20200183.

Costa, R. E. A. R. da, Paulo, S. A., Vieira, A. L., Balsamo, A. C. M., Brito, A. C. S., Mesquita, M. M. de, Souza, D. B., Souza, I. L. de, Pedroso, A. L. de O., Müller, P. C. T., Rodrigues, K. S., Leal, T. L. S. L., Sipaúba, T. S., Mendes, A. M., Barroso, I. D., \& Torres, I. L. (2020). Principais correlações do tabagismo com a COVID-19. Research, Society and Development, 9(9), e84996981. https://doi.org/10.33448/rsd-v9i9.6981

Emami, A., Javanmardi, F., Pirbonyeh, N., \& Akbari, A. (2020). Prevalence of Underlying Diseases in Hospitalized Patients with COVID-19: a Systematic Review and Meta-Analysis. Archives of academic emergency medicine, 8(1), e35.

Faria, D. A. de, \& Fonseca, P. H. N. da. (2021). WhatsApp® como Recurso para a Educação em Saúde: Acompanhamento de grupo de cessação do tabagismo diante da pandemia da COVID-19. Research, Society and Development, 10(7), e2910716166. https://doi.org/10.33448/rsd-v10i7.16166

González Hidalgo, J. A., Díaz Piedra, J. A., Alfonso Díaz, K., \& Avalos González, M. M. (2012). Hábito de fumar en la adolescencia. Revista Cubana de Medicina General Integral, 28(3), 282-289.

Gülsen, A., Yigitbas, B. A., Uslu, B., Drömann, D., \& Kilinc, O. (2020). The Effect of Smoking on COVID-19 Symptom Severity: Systematic Review and Meta-Analysis. Pulmonary medicine, 2020, 7590207. https://doi.org/10.1155/2020/7590207

Hamet, P., Pausova, Z., Attaoua, R., Hishmih, C., Haloui, M., Shin, J., Paus, T., Abrahamowicz, M., Gaudet, D., Santucci, L., Kotchen, T. A., Cowley, A. W., Hussin, J., \& Tremblay, J. (2021). SARS-CoV-2 Receptor ACE2 Gene Is Associated with Hypertension and Severity of COVID 19: Interaction with Sex, Obesity, and Smoking. American journal of hypertension, 34(4), 367-376. https://doi.org/10.1093/ajh/hpaa223

INCA. Instituto Nacional de Câncer José Alencar Gomes da Silva. (2020). Perguntas frequentes: Quem fuma faz parte do grupo de risco para o coronavírus (Covid-19)? https://www.inca.gov.br/perguntas-frequentes/tabagismo-e-coronavirus-covid-19\#: :text=Quem\%20fuma\%20faz\%20parte\%20d o,por\%20v\%C $3 \%$ A Drus \%2C\%20bact\%C3\%A9rias\%20e\%20fungos. Acesso: 18 julho 2021

Jackson, S. E., Garnett, C., Shahab, L., Oldham, M., \& Brown, J. (2021). Association of the COVID-19 lockdown with smoking, drinking and attempts to quit in England: an analysis of 2019-20 data. Addiction (Abingdon, England), 116(5), 1233-1244. https://doi.org/10.1111/add.15295

Komiyama, M., \& Hasegawa, K. (2020). Smoking Cessation as a Public Health Measure to Limit the Coronavirus Disease 2019 Pandemic. European cardiology, 15, e16. https://doi.org/10.15420/ecr.2020.11

Liu, W., Tao, Z. W., Wang, L., Yuan, M. L., Liu, K., Zhou, L., Wei, S., Deng, Y., Liu, J., Liu, H. G., Yang, M., \& Hu, Y. (2020). Analysis of factors associated with disease outcomes in hospitalized patients with 2019 novel coronavirus disease. Chinese medical journal, 133(9), 1032-1038. https://doi.org/10.1097/CM9.0000000000000775

Melo, L. D. de, Jeremias, J. da S., Shubo, A. F. M. F., Taroco, F. E., Spindola, T., Gomes Filho, W., Ferreira, B. S. B., \& Barbosa, D. J. (2020). Tabagismo, Hipertensão Arterial Sistêmica e Pandemia da COVID-19: uma Análise Psicanalítica Freudiana. Research, Society and Development, 9(11), e57891110240. https://doi.org/10.33448/rsd-v9i11.10240

OPAS. Organização Pan-Americana de Saúde (2020). Folha informativa COVID-19 - Escritório da OPAS e da OMS no Brasil. https://www.paho.org/pt/covid19. Acesso: 18 julho 2021.

Pessoa, D. L. R., Santos, K. F. dos, Rodrigues, K. P., Dias, A. M. C., Souza, I. B. J. de, Gouveia, D. M. de, \& Ramos, A. S. M. B. (2020). Reflexões sobre a associação entre o tabagismo e COVID - 19: revisão de literatura. Research, Society and Development, 9(9), e455997450. https://doi.org/10.33448/rsdv9i9.7450

Piras, S. S., Latuf, G. M. D. O., Pereira, A. C. E. D. S., Elias, F. T. S., \& Camargo, E. B. (2020). Electronic cigarette use and smoking initiation in adolescents and young adults: evidence synthesis. Comunicação em Ciências da Saúde, Brasília, 31(2), 75-82.

Rodrigues, G. M. C., Silva, A. R. C., \& Rodrigues, A. P. (2020). Agravos da infecção por COVID-19 em usuários do tabaco. Revista de Iniciação Científica da Libertas, 10(1), 112.

Rodrigues, L. A., Rodrigues, A. D. F. M., Patrício, J. A. L., \& Breda, I. S. (2020). A relação entre o tabagismo e a hospitalização por COVID-19: revisão integrativa. Unesc em Revista, 4(2), 99-113.

Salem Szklo, A. (2020). Associação entre Fumar e Progressão para Complicações Respiratórias Graves em Pacientes com Covid-19. Revista Brasileira De Cancerologia, 66(2), e-03974. https://doi.org/10.32635/2176-9745.RBC.2020v66n2.974

Scholz, J. R., Lopes, M. A. C. Q., Saraiva, J. F. K., \& Colombo, F. C. (2020). COVID-19, Sistema Renina-Angiotensina, Enzima Conversora da Angiotensina 2 e Nicotina: Qual a Inter-Relação? Arquivos Brasileiros de Cardiologia, 115(4), 708-711. https://doi.org/10.36660/abc.20200653

Silva, A. L. O. D., Moreira, J. C., \& Martins, S. R. (2020). COVID-19 e tabagismo: uma relação de risco. Cadernos de Saúde Pública, 36(5). https://doi.org/10.1590/0102-311X00072020

Sokolovsky, A. W., Hertel, A. W., Micalizzi, L., White, H. R., Hayes, K. L., \& Jackson, K. M. (2021). Preliminary impact of the COVID-19 pandemic on smoking and vaping in college students. Addictive behaviors, 115, 106783. https://doi.org/10.1016/j.addbeh.2020.106783

van Zyl-Smit, R. N., Richards, G., \& Leone, F. T. (2020). Tobacco smoking and COVID-19 infection. The Lancet. Respiratory medicine, 8(7), 664-665. https://doi.org/10.1016/S2213-2600(20)30239-3 
Research, Society and Development, v. 10, n. 10, e222101018442, 2021

(CC BY 4.0) | ISSN 2525-3409 | DOI: http://dx.doi.org/10.33448/rsd-v10i10.18442

WHO. World Health Organization. (2020a). Q\&A: Tobacco and COVID-19 [Internet]. WHO: https://www.who.int/emergencies/diseases/novel-coronavirus2019/question-and-answers-hub/q-a-detail/q-a-on-smoking-and-covid-19.

WHO. World Health Organization. (2020b). Tobacco Free Initiative. Tobacco and waterpipe use increase the risk of COVID-19. http://www.emro.who.int/tfi/know-the-truth/tobacco-and-waterpipe-users-are-at-increased-risk-of-covid-19-infection.html. 